\title{
Constraints on the CP-Violating MSSM ${ }^{\text {« }}$
}

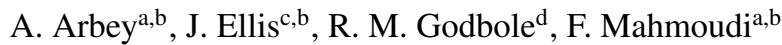 \\ ${ }^{a}$ Univ Lyon, Univ Lyon 1, ENS de Lyon, CNRS, Centre de Recherche Astrophysique de Lyon UMR5574, F-69230 Saint-Genis-Laval, France \\ ${ }^{b}$ Theoretical Physics Department, CERN, CH-1211 Geneva 23, Switzerland \\ ${ }^{c}$ Theoretical Particle Physics and Cosmology Group, Department of Physics, King's College London, London WC2R 2LS, United Kingdom \\ ${ }^{d}$ Centre for High Energy Physics, Indian Institute of Science, Bangalore, 560012, India
}

\begin{abstract}
We discuss the prospects for observing CP violation in the MSSM with six CP-violating phases, using a geometric approach to maximise CP-violating observables subject to the experimental upper bounds on electric dipole moments. We consider constraints from Higgs physics, flavour physics, the dark matter relic density and spin-independent scattering cross section with matter.
\end{abstract}

Keywords: CP violation, supersymmetry

\section{Introduction}

In the minimal supersymmetric extension of the Standard Model (MSSM), there are possibilities of having new sources of $\mathrm{CP}$ violation beyond the Cabibbo Kobayashi Maskawa (CKM) phase. However, experimental constraints in particular from the measurements of electric dipole moments (EDMs, see Tab. 1) limit strongly the values of the additional phases. Here we consider the maximally CP-violating, minimally flavour-violating (MCPMFV) model that contains 6 new CP-violating phases: 3 phases $\Phi_{1,2,3}$ in the masses of the U(1), SU(2) and SU(3) gauginos, and 3 phases $\Phi_{t, b, \tau}$ in the trilinear soft supersymmetry breaking couplings $A_{t, b, \tau}$ of the third-generation stop, sbottom and stau sfermions, respectively. Brute force to sample randomly the values of the phases imposing all the experimental constraints (from Higgs, flavour physics, dark matter and EDMs) appears to be inefficient and one would need an optimised procedure such as a geometric approach [1, 2]. We study signatures of CP violation considering the lightest neutral Higgs boson to be

\footnotetext{
${ }^{2}$ Based on the talk by A.A. at the Sixth Workshop on Theory, Phenomenology and Experiments in Flavour Physics, Capri, June 2016.

Email address: alexandre.arbey@ens-lyon.fr (A. Arbey)
}

\begin{tabular}{|c|c|c|}
\hline EDM & Upper limit (e.cm) & Reference \\
\hline \hline Thallium & $1.3 \times 10^{-24}$ & {$[3]$} \\
\hline Mercury & $3.5 \times 10^{-29}$ & {$[4]$} \\
\hline Neutron & $4.7 \times 10^{-26}$ & {$[5]$} \\
\hline Thorium monoxide & $1.1 \times 10^{-28}$ & {$[6]$} \\
\hline
\end{tabular}

Table 1: 95\% CL upper limits on the EDMs used in this study.

the observed one and the lightest supersymmetric particle to be the lightest neutralino, and study in particular the $\mathrm{CP}$ asymmetry in $b \rightarrow s \gamma, B_{s}$ meson mixing $\Delta M_{B_{s}}$, and CP-violating couplings of the heavier neutral Higgs bosons in several representative MSSM scenarios. Extended discussions can be found in [2].

\section{Methodology}

We consider here two different MSSM scenarios: the CPV-CMSSM and the CPV-pMSSM, which are extensions of the standard CMSSM and pMSSM scenarios incorporating six additional phases $\Phi_{1,2,3, t, b, \tau}$ to account 
for $\mathrm{CP}$ violation. We perform flat random scans on the standard parameters, similarly to [7- -9$]$. The phases however are very severely constrained by the electric dipole moment (EDM) measurements given in Tab. 1 so that the use of an optimised geometric technique becomes necessary. The muon EDM has not been used in our analysis since its experimental upper bound [10] only provides very weak constraints that are not competitive with the constraints from the other EDMs.

Let us consider the four EDMs, $E^{a, b, c, d}$, of Tab. 1 in the small phase approximation, with

$$
E^{i} \simeq \boldsymbol{\Phi} \cdot \mathbf{E}^{i},
$$

where $\boldsymbol{\Phi} \equiv \Phi_{\alpha}=\Phi_{1,2,3, t, b, \tau}$ and $\mathbf{E}^{i} \equiv \partial E^{i} / \partial \boldsymbol{\Phi}$, and an additional CP-violating observable $O$ in the the small phase approximation, such that

$$
\mathbf{O} \equiv \partial O / \partial \boldsymbol{\Phi} .
$$

The optimal direction that maximises the observable $O$ and is orthogonal to the EDM vectors $E_{\alpha}^{a, b, c, d}$ is given by

$$
\Phi_{\alpha}=\epsilon_{\alpha \beta \gamma \delta \mu \eta} \epsilon_{\eta \nu \lambda \rho \sigma \tau} E_{\beta}^{a} E_{\gamma}^{b} E_{\delta}^{c} E_{\mu}^{d} O_{\nu} E_{\lambda}^{a} E_{\rho}^{b} E_{\sigma}^{c} E_{\tau}^{d},
$$

with an unknown normalisation factor.

For each choice of the CP-conserving parameters, we fix the phases to $0^{\circ}$ or $\pm 180^{\circ}$, and compute the optimal direction using Eq. (3). Sets of phases are chosen randomly along this direction, then moved by $20^{\circ}$ along the favoured direction. Subsequently, the favoured direction is recomputed at this new position. We iterate this procedure up to $100^{\circ}$.

The SUSY mass spectra and couplings, and EDM constraints are computed with CPsuperH [11-13]. The thorium monoxide EDM is computed following [14]. Flavour observables are calculated with SuperIso [15, 16] and CPsuperH. The dark matter relic density is computed with SuperIso Relic [17] and micrOMEGAs [18], and the later is also used to compute dark matter direct detection observables. Finally, HiggsBounds [19] is used to impose the Higgs constraints. In contrast to the CP-conserving MSSM scenarios, here the couplings and mixing matrices can be complex, and the three Higgs bosons can also be mixed and have scalar and pseudoscalar components, leading to three states $h_{1,2,3}$, named in the order of increasing masses.

\section{Constraints in the CMSSM}

We consider the CMSSM as a benchmark scenario to study the effects of the CP phases. For this purpose, we focus on the CP-conserving CMSSM best-fit point found in a global analysis [20]:
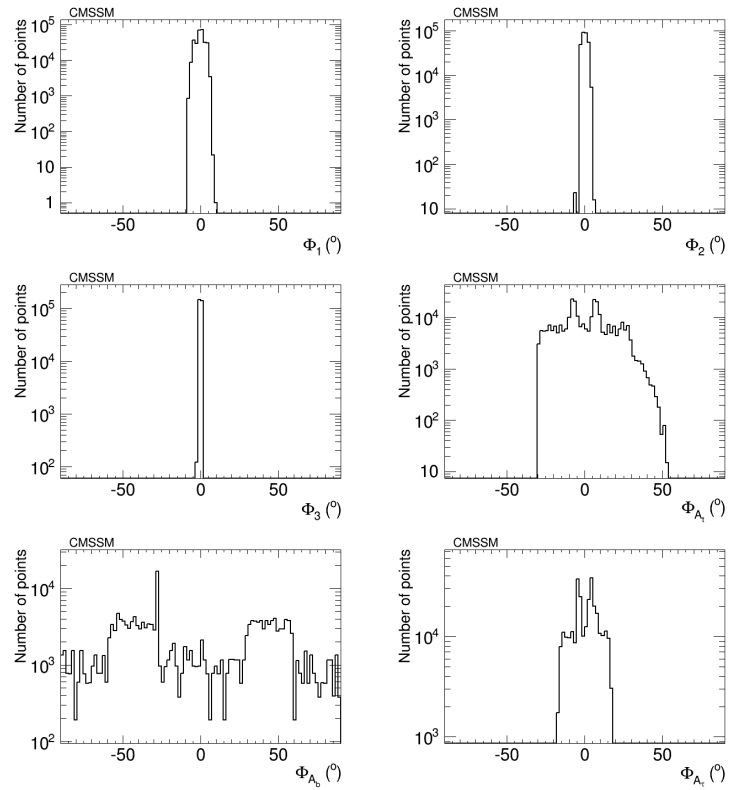

Figure 1: Number of CMSSM points allowed by the EDM constraints, starting from a flat distribution, as a function of the phases.

$$
\begin{gathered}
m_{0}=670 \mathrm{GeV}, m_{1 / 2}=1040 \mathrm{GeV}, A_{0}=3440 \mathrm{GeV}, \\
\tan \beta=21,
\end{gathered}
$$

and vary the phases subsequently. To test the efficiency of the geometric approach, we vary the phases randomly, then employ the geometric approach and finally apply the constraints. 600.000 points are generated for each case. Imposing the constraints removes about $85 \%$ of the points in the purely random approach, while only $50 \%$ of the points are removed with the geometric approach, showing the efficiency of the latter.

Fig. 1 shows the distribution of the phases obtained by imposing the EDM constraints. Clearly, the phases $\Phi_{1}, \Phi_{2}, \Phi_{3}$ are strongly constrained. The EDM constraints also severely restrict the possible Higgs masses, as shown in Fig. 2. In addition, the EDM constraints impose the Higgs signal strengths to be very close to 1, as demonstrated in Fig. 3 .

More generically, when varying all the CMSSM parameters, the EDM measurements strongly limit the CPviolating CMSSM to be very close to the $\mathrm{CP}$-conserving CMSSM (see [2] for more details). This conclusion however does not hold in an unconstrained scenario such as the phenomenological MSSM (pMSSM). 

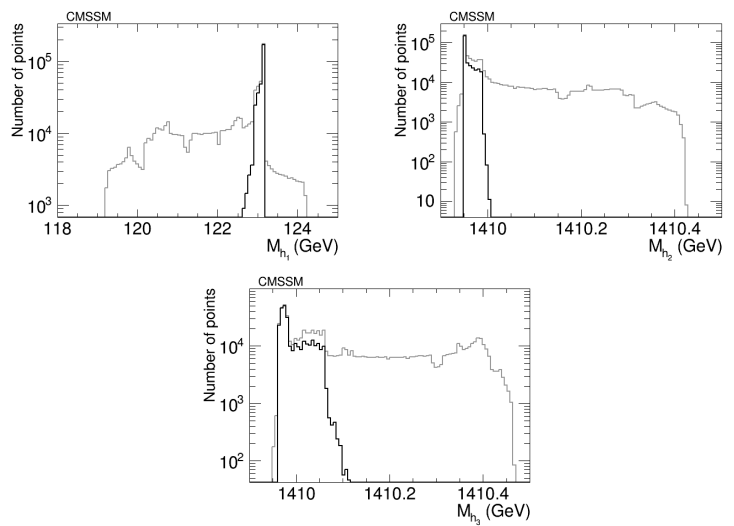

Figure 2: Distribution of the Higgs masses for the considered CMSSM scenario. The gray line corresponds to the number of points before applying the EDM constraints, and the black one after imposing them.
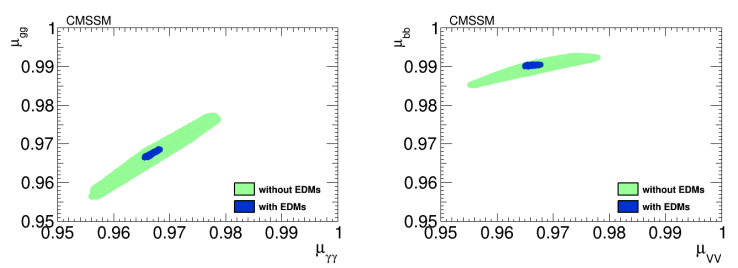

Figure 3: Lighter Higgs signal strengths in $\left(\mu_{\gamma \gamma}, \mu_{g g}\right)$ (left panel) and $\left(\mu_{V V}, \mu_{b b}\right)$ (right panel) planes before and after imposing the EDM constraints.

\section{Constraints in the pMSSM}

To study the pMSSM, we first vary the $19 \mathrm{CP}$ conserving pMSSM parameters. We then use the geometric approach to choose points with phases between $-180^{\circ}$ and $+180^{\circ}$. 40 million such points have been generated with gluino and squark masses compatible with the LHC limits. We then impose the $h_{1}$ mass to lie between 121 and $129 \mathrm{GeV}$ and the lightest neutralino to be the lightest supersymmetric particle so that it constitutes a dark matter candidate. This reduces the number of points to about one million. After imposing the EDM constraints, we are left with 15.000 points. The distribution of the CP phases is shown in Fig. 4

As can be seen, the $\Phi_{2}$ phase is particularly constrained, and the other phases can take any values. In Fig. 5, the phases of the points compatible with the EDM constraints are drawn in 2 dimensional plots. They reveal that the EDM constraints impose correlations between the phases related to the gluino and thirdgeneration squarks, with prefered directions that are revealed by the geometric approach.

In the following, we study the influence of $\mathrm{CP}$ viola-
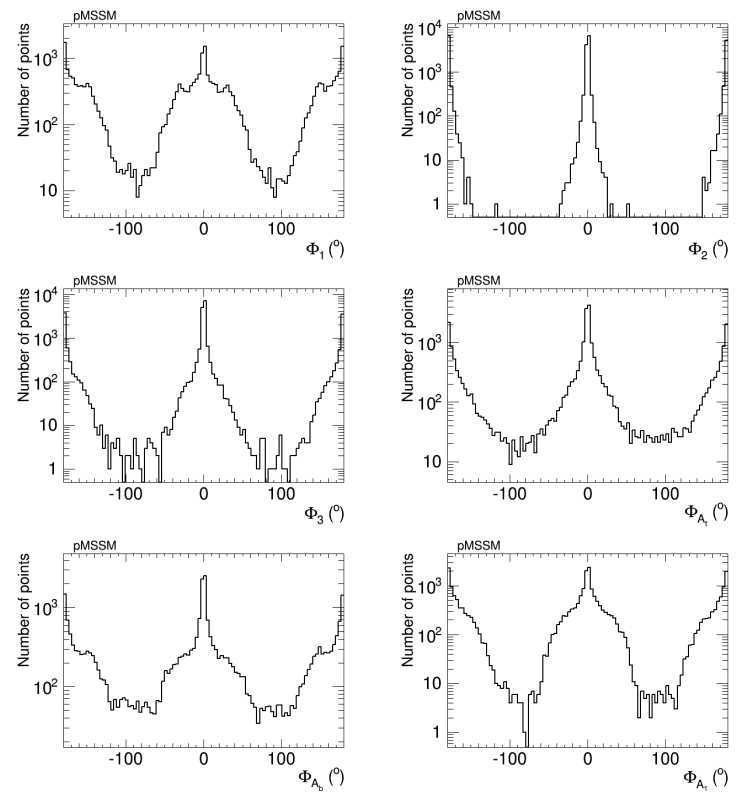

Figure 4: Number of pMSSM points allowed by the EDM constraints as a function of the phases.
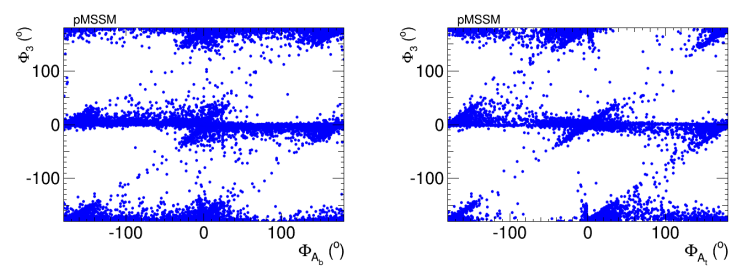

Figure 5: pMSSM points surviving the EDM constraints in the $\left(\Phi_{b}, \Phi_{3}\right)$ (left panel) and $\left(\Phi_{t}, \Phi_{3}\right)$ (right panel) parameter planes.

tion on the dark matter, Higgs and flavour sectors.

\subsection{Dark matter sector}

We first compute the neutralino relic density and compare the results to the cosmological cold dark matter density $\Omega_{c} h^{2} \sim 0.11$ [21]. The results are presented in the upper panel of Fig. 6. We notice that the points with $\mathrm{CP}$ conservation and the ones with $\mathrm{CP}$ violation are similarly spread, such that the $\mathrm{CP}$ phases do not add much content to the picture. The points above the cosmological measurements have mostly bino-like neutralino, and the two strips below the dark matter line correspond to wino and higgsino-like neutralinos. The points above the line can be considered as excluded, since they lead to an overdensity of dark matter, provided the pre-Big Bang nucleosynthesis period is dominated by radiation [22, 23], so that most of the bino-like neutralinos are excluded. CP violation allows for more such points to sur- 

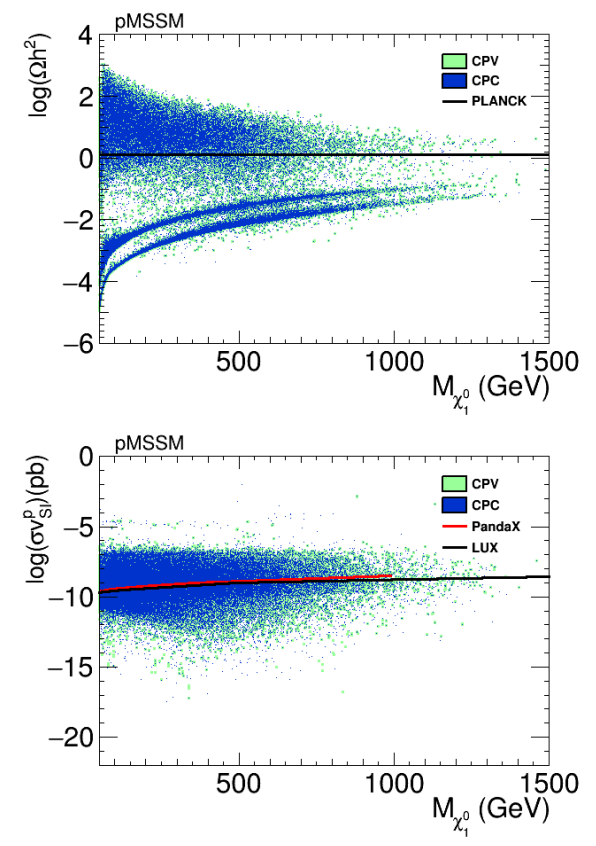

Figure 6: pMSSM points compatible with the EDM constraints in the relic density vs. neutralino 1 mass (upper panel) and neutralino spin-independent scattering cross section with proton vs. neutralino 1 mass (lower panel). The blue points are the points without $\mathrm{CP}$ violation, and the green points have $\mathrm{CP}$-violating phases. The black line on the upper plot corresponds to the central value of the dark matter density measured with cosmological observations, and the red and black curves on the lower plot to the PandaX and LUX 2016 upper limits on the scattering cross section.

vive, without changing the picture. For the points below the line the neutralino relic density could only partially account for the whole dark matter density, and they can therefore be considered as still allowed.

We also consider dark matter direct detection and the latest upper limits provided by the PandaX [24] and LUX [25] experiments. The results are shown in the lower panel of Fig. 6. Similarly to the case of the relic density, we see that while $\mathrm{CP}$ violation allows for some more spread of the points in comparison to the $\mathrm{CP}$-conserving case, it does not add much to the picture. This shows that dark matter observables are unlikely to disentangle CP-violating scenarios form the $\mathrm{CP}$-conserving ones.

\subsection{Higgs sector}

First, we assume the lightest Higgs boson to be the one discovered at the LHC by imposing its mass to lie between 121 and $129 \mathrm{GeV}$, so as to allow for theoretical uncertainties in its calculation, and we study the modifications to the signal strengths due to $\mathrm{CP}$ violation. The
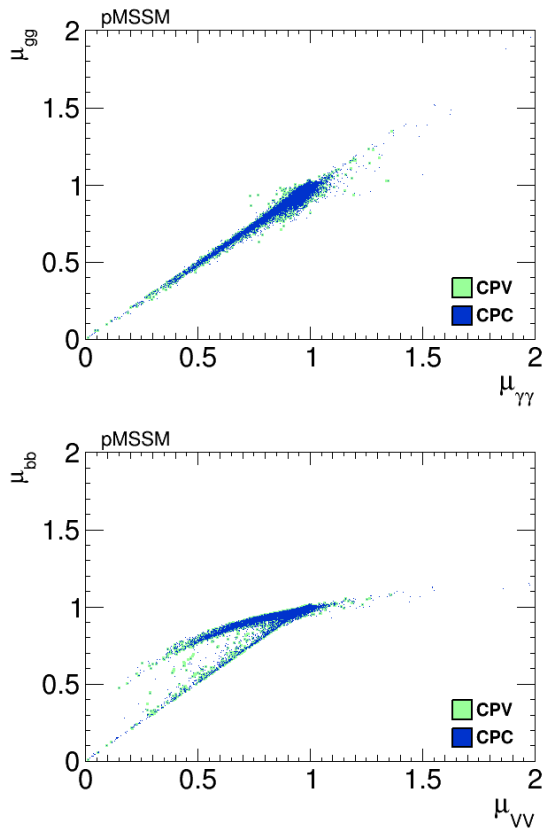

Figure 7: pMSSM points compatible with the EDM constraints in the $\mu_{g g}$ vs. $\mu_{\gamma \gamma}$ (upper panel) and $\mu_{b b}$ vs. $\mu_{V V}$ (lower panel) parameter planes. The blue points are the points without $\mathrm{CP}$ violation, and the green points have $\mathrm{CP}$-violating phases.

signal strength in the decay channel $h_{1} \rightarrow X X$ is defined as the ratio of the cross section production of the $h_{1}$ times its decay branching ratio to $X X$ over the Standard Model values. The LHC results for the measured decay modes show that the signal strengths are compatible with 1 [26]. Our results are summarised in Fig. 7. As can be seen, the set of points with $\mathrm{CP}$ violation are not distinct from the one with $\mathrm{CP}$ conservation, so that the signal strength measurement will not allow to probe the difference between the two cases.

The Higgs sector however provides other observables which can allow to probe $\mathrm{CP}$ violation. The Higgs bosons can be admixtures of scalar and pseudoscalar components. On the one side, the measurements of the $h_{1}$ properties showed that it is mostly a scalar, and the pseudoscalar component could only be tiny, and adding the EDM constraints limits the pseudoscalar component to be negligible. On the other side, the two heavier Higgses can be mixtures of scalar and pseudoscalar components. We denote $g_{S, P}^{h_{i} \bar{f} f}$ the scalar and pseudoscalar couplings of $h_{i}$ to $\bar{f} f$ and define

$$
\tan \phi_{\tau}^{h_{i}} \equiv \frac{g_{P}^{h_{i} \tau \tau}}{g_{S}^{h_{i} \tau \tau}}, \quad \tan \phi_{t}^{h_{i}} \equiv \frac{g_{P}^{h_{i} \bar{t} t}}{g_{S}^{h_{i} t}}
$$



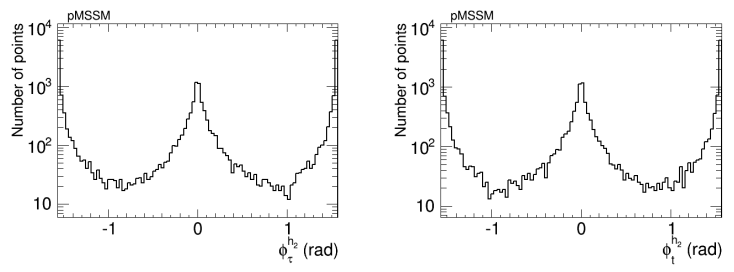

Figure 8: Distribution of the $\phi_{\tau}^{h_{2}}$ and $\phi_{t}^{h_{2}}$ angles in the CPV-pMSSM after imposing the EDM constraints.

The angles $\phi_{\tau}^{h_{i}}$ and $\phi_{t}^{h_{i}}$ characterise the scalar and pseudoscalar couplings to the $\tau$ leptons and top quarks, respectively. An angle close to 0 means a mostly scalar coupling, while $\pi / 2$ means a pure pseudoscalar coupling. In Fig. 8, we show the distributions of the angles of the $h_{2}$ after imposing the EDM constraints. Similar plots can be obtained for $h_{3}$ [2]. These results demonstrate that strongly-mixed states are still allowed for the heavy Higgses, and that a measurement of the $\tau \tau$ and $t \bar{t}$ spin correlations in $h_{2,3}$ decays may reveal the CPviolating nature of the MSSM.

\subsection{Flavour sector}

$\mathrm{CP}$ violation is intimately related to flavour physics, in particular because of the CP-violating phase present in the CKM matrix. Flavour-changing neutral currents constitute important observables to probe for new physics and $\mathrm{CP}$ violation. In particular, the inclusive decay $B \rightarrow X_{s} \gamma$ measured by $B$-factories provides valuable constraints. In Fig. 9, we present the constraints imposed by the measurements of the branching ratio and CP asymmetry of this decay on our PMSSM points passing the EDM constraints. For comparison, we show the current [27] and prospective [28] limits. As can be seen, the current limits are superseded by the EDM constraints, but the perspectives to discover $\mathrm{CP}$ violation in the MSSM at Belle-II are promising.

Another important flavour observable is the $B_{S}$ meson mixing $\Delta M_{B_{s}}$. As can be seen in Fig. 10, the CPviolating MSSM contributions to $\Delta M_{B_{S}}^{N P}$ are in general below the present upper limit, which is dominated by theoretical uncertainties. If these could be reduced, $\Delta M_{B_{s}}$ could also provide an interesting and complementary constraint on the phases, enabling them to be determined experimentally, in principle.

\section{Conclusion}

We have explored the effects of CP violation in the MSSM using an iterative geometrical approach within
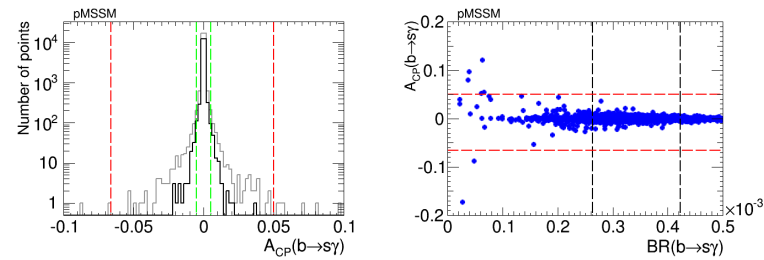

Figure 9: In the left panel, the distributions of the values of the CP asymmetry of $B \rightarrow X_{s} \gamma$ for the pMSSM points are displayed. The gray curve corresponds to the points before the EDM constraints, and the black curve after the EDM constraints. The red dashed lines correspond to the current limits and the green dashed lines to the prospective Belle-II limits. In the right panel, the pMSSM points passing the EDM constraints in the CP asymmetry vs. branching ratio of $B \rightarrow X_{s} \gamma$ parameter plane are shown. The lines correspond to the current limits.

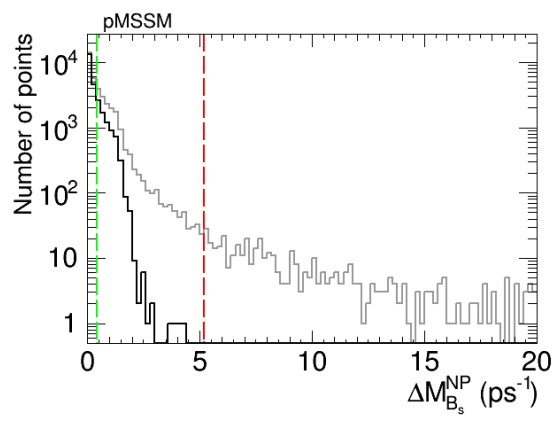

Figure 10: Distribution of the values of $\Delta M_{B}^{N P}$ for the pMSSM points. The gray curve corresponds to the points before the EDM constraints, and the black curve after the EDM constraints. The red dashed line shows the current limit and the green dashed line the prospective limit after a factor 10 improvement in the form factor determination.

the maximally CP-violating, minimal flavour-violating framework with $6 \mathrm{CP}$-violating phases, applying constraints from the Higgs mass and signal strengths, flavour physics, dark matter relic density and scattering cross section with matter.

In the CMSSM scenario, we found relatively little scope for large deviations from the $\mathrm{CP}$-conserving case, e.g., in the masses of the Higgs bosons and the spinindependent dark matter scattering cross section. Moreover, we found that only very small values of $A_{C P}$ would be possible in this case, and the new physics contribution to $B_{s}$ meson mixing would not be observable.

In the pMSSM scenario, the $A_{C P}$ could be as large as $\sim 3 \%$, within the reach of the Belle-II experiment. We found in this scenario that $\Delta M_{B_{s}}^{N P}$ could be large enough to be observable with a prospective reduction in the theoretical uncertainty in the Standard Model calculation of $B_{s}$ mixing.

The CP-violating phases in the $h_{1} \tau^{+} \tau^{-}$and $h_{1} \bar{t} t$ cou- 
plings are in general small in the MSSM. However, the phases in the $h_{2,3} \tau^{+} \tau^{-}$and $h_{2,3} \bar{t} t$ couplings can be quite large, and may present interesting prospects for future experiments.

In general, we showed that the EDM constraints do not force all the six CP-violating phases to be small, and in some variants of the MSSM there could be observable signatures of $\mathrm{CP}$ violation beyond the Standard Model, such as $A_{C P}$ in the $B \rightarrow X_{s} \gamma$ decay and $\Delta M_{B_{s}}^{N P}$.

\section{Acknowledgements}

A.A. and F.M. would like to thank the organisers for their invitation and the fruitful workshop, and would like to express a special thank to the Mainz Institute for Theoretical Physics (MITP), the Università di Napoli Federico II and INFN for their hospitality and support.

\section{References}

[1] J. Ellis, J. S. Lee, A. Pilaftsis, A Geometric Approach to CP Violation: Applications to the MCPMFV SUSY Model, JHEP 10 (2010) 049. arXiv:1006.3087, doi:10.1007/ JHEP10 (2010) 049

[2] A. Arbey, J. Ellis, R. M. Godbole, F. Mahmoudi, Exploring CP Violation in the MSSM, Eur. Phys. J. C75 (2) (2015) 85. arXiv: 1410.4824 doi:10.1140/epjc/s10052-015-3294-z

[3] B. C. Regan, E. D. Commins, C. J. Schmidt, D. DeMille, New limit on the electron electric dipole moment, Phys. Rev. Lett. 88 (2002) 071805. doi:10.1103/PhysRevLett.88.071805

[4] W. C. Griffith, M. D. Swallows, T. H. Loftus, M. V. Romalis, B. R. Heckel, E. N. Fortson, Improved Limit on the Permanent Electric Dipole Moment of Hg-199, Phys. Rev. Lett. 102 (2009) 101601. doi:10.1103/PhysRevLett.102.101601

[5] C. A. Baker, et al., An Improved experimental limit on the electric dipole moment of the neutron, Phys. Rev. Lett. 97 (2006) 131801. arXiv:hep-ex/0602020 doi:10.1103/ PhysRevLett.97.131801

[6] J. Baron, et al., Order of Magnitude Smaller Limit on the Electric Dipole Moment of the Electron, Science 343 (2014) 269272. arXiv: 1310.7534 doi:10.1126/science.1248213

[7] A. Arbey, M. Battaglia, F. Mahmoudi, Implications of LHC Searches on SUSY Particle Spectra: The pMSSM Parameter Space with Neutralino Dark Matter, Eur. Phys. J. C72 (2012) 1847. arXiv:1110.3726 doi:10.1140/epjc/ s10052-011-1847-3

[8] A. Arbey, M. Battaglia, F. Mahmoudi, Constraints on the MSSM from the Higgs Sector: A pMSSM Study of Higgs Searches, $B_{s}^{0} \rightarrow \mu^{+} \mu^{-}$and Dark Matter Direct Detection, Eur. Phys. J. C72 (2012) 1906. arXiv:1112.3032 doi:10.1140/epjc/ s10052-012-1906-4

[9] A. Arbey, M. Battaglia, A. Djouadi, F. Mahmoudi, An update on the constraints on the phenomenological MSSM from the new LHC Higgs results, Phys. Lett. B720 (2013) 153-160. arXiv : 1211.4004 doi:10.1016/j.physletb.2013.02.001

[10] G. W. Bennett, et al., An Improved Limit on the Muon Electric Dipole Moment, Phys. Rev. D80 (2009) 052008. arXiv: 0811. 1207 doi:10.1103/PhysRevD.80.052008

[11] J. S. Lee, A. Pilaftsis, M. Carena, S. Y. Choi, M. Drees, J. R. Ellis, C. E. M. Wagner, CPsuperH: A Computational tool for Higgs phenomenology in the minimal supersymmetric standard model with explicit CP violation, Comput. Phys. Commun. 156 (2004) 283-317. arXiv:hep-ph/0307377. doi: 10.1016/S0010-4655(03) 00463-6
[12] J. S. Lee, M. Carena, J. Ellis, A. Pilaftsis, C. E. M. Wagner, CPsuperH2.0: an Improved Computational Tool for Higgs Phenomenology in the MSSM with Explicit CP Violation, Comput. Phys. Commun. 180 (2009) 312-331. arXiv:0712 .2360 doi:10.1016/j.cpc.2008.09.003

[13] J. S. Lee, M. Carena, J. Ellis, A. Pilaftsis, C. E. M. Wagner, CPsuperH2.3: an Updated Tool for Phenomenology in the MSSM with Explicit CP Violation, Comput. Phys. Commun. 184 (2013) 1220-1233. arXiv:1208.2212 doi:10.1016/ j.cpc.2012.11.006

[14] K. Cheung, J. S. Lee, E. Senaha, P.-Y. Tseng, Confronting Higgcision with Electric Dipole Moments, JHEP 06 (2014) 149. arXiv: 1403.4775 doi:10.1007/JHEP06(2014) 149

[15] F. Mahmoudi, SuperIso: A Program for calculating the isospin asymmetry of $B \rightarrow K^{*} \gamma$ in the MSSM, Comput. Phys. Commun. 178 (2008) 745-754. arXiv:0710.2067 doi:10.1016/j. cpc. 2007.12.006

[16] F. Mahmoudi, SuperIso v2.3: A Program for calculating flavor physics observables in Supersymmetry, Comput. Phys. Commun. 180 (2009) 1579-1613. arXiv:0808.3144 doi:10. $1016 / j \cdot c p c .2009 .02 .017$

[17] A. Arbey, F. Mahmoudi, SuperIso Relic: A Program for calculating relic density and flavor physics observables in Supersymmetry, Comput. Phys. Commun. 181 (2010) 1277-1292. arXiv:0906.0369 doi:10.1016/j.cpc.2010.03.010

[18] G. Belanger, F. Boudjema, A. Pukhov, A. Semenov, micrOMEGAs 3: A program for calculating dark matter observables, Comput. Phys. Commun. 185 (2014) 960-985. arXiv: 1305.0237 doi:10.1016/j.cpc.2013.10.016

[19] P. Bechtle, O. Brein, S. Heinemeyer, O. Stal, T. Stefaniak, G. Weiglein, K. E. Williams, HiggsBounds - 4: Improved Tests of Extended Higgs Sectors against Exclusion Bounds from LEP, the Tevatron and the LHC, Eur. Phys. J. C74 (3) (2014) 2693. arXiv:1311.0055 doi:10.1140/ epjc/s10052-013-2693-2

[20] O. Buchmueller, et al., The CMSSM and NUHM1 after LHC Run 1, Eur. Phys. J. C74 (6) (2014) 2922. arXiv:1312.5250 doi:10.1140/epjc/s10052-014-2922-3

[21] P. A. R. Ade, et al., Planck 2015 results. XIII. Cosmological parameters, Astron. Astrophys. 594 (2016) A13. arXiv: 1502. 01589 doi: 10.1051/0004-6361/201525830

[22] A. Arbey, F. Mahmoudi, SUSY constraints from relic density: High sensitivity to pre-BBN expansion rate, Phys. Lett. B669 (2008) 46-51. arXiv:0803.0741 doi:10.1016/j. physletb.2008.09.032

[23] A. Arbey, F. Mahmoudi, SUSY Constraints, Relic Density, and Very Early Universe, JHEP 05 (2010) 051. arXiv : 0906.0368 doi:10.1007/JHEP05(2010)051

[24] A. Tan, et al., Dark Matter Results from First 98.7 Days of Data from the PandaX-II Experiment, Phys. Rev. Lett. 117 (12) (2016) 121303. arXiv: 1607.07400 doi:10.1103/ PhysRevLett.117.121303

[25] D. S. Akerib, et al., Results from a search for dark matter in the complete LUX exposure arXiv: 1608.07648

[26] G. Aad, et al., Measurements of the Higgs boson production and decay rates and constraints on its couplings from a combined ATLAS and CMS analysis of the LHC pp collision data at $\sqrt{s}=$ 7 and 8 TeV, JHEP 08 (2016) 045. arXiv: 1606.02266 doi: 10.1007/JHEP08(2016) 045

[27] C. Patrignani, et al., Review of Particle Physics, Chin. Phys. C40 (10) (2016) 100001. doi:10.1088/1674-1137/40/10/ 100001

[28] T. Aushev, et al., Physics at Super B FactoryarXiv:1002. 5012 